\title{
On the Algebraic Index for Riemannian Étale Groupoids
}

\author{
MARKUS J. PFLAUM ${ }^{1}$, HESSEL POSTHUMA ${ }^{2}$ and XIANG TANG ${ }^{3}$ \\ ${ }^{1}$ Department of Mathematics, University of Colorado, Boulder, USA. \\ e-mail:markus.pflaum@colorado.edu \\ ${ }^{2}$ Korteweg-de Vries Institute for Mathematics, University of Amsterdam, Amsterdam, \\ The Netherlands.e-mail: H.B.Posthuma@uva.nl \\ ${ }^{3}$ Department of Mathematics, Washington University, St. Louis, USA. \\ e-mail: xtang@math.wustl.edu
}

Received: 22 December 2008 / Revised: 3 July 2009 / Accepted: 3 July 2009

Published online: 1 October 2009 - (C) The Author(s) 2009. This article is published with open access at Springerlink.com

\begin{abstract}
In this paper, we construct an explicit quasi-isomorphism to study the cyclic cohomology of a deformation quantization over a Riemannian étale groupoid. Such a quasi-isomorphism allows us to propose a general algebraic index problem for Riemannian étale groupoids. We discuss solutions to that index problem when the groupoid is proper or defined by a constant Dirac structure on a 3-dimensional torus.
\end{abstract}

Mathematics Subject Classification (2000). Primary 58J20; Secondary 53D55.

Keywords. Riemannian foliation, deformation quantization, index, cyclic cohomology.

\section{Introduction}

In [17] and [18], the authors of this paper studied the algebraic index theory over orbifolds using noncommutative geometry and deformation quantization. In [17], we obtained an explicit topological formula for the Chern character of an elliptic operator on a compact Riemannian orbifold. With this paper we continue our study of algebraic index theory over singular spaces modeled by groupoids. More precisely, omitting the assumption of properness from [17] and [18] we study here the algebraic index theory of a singular space which can be obtained as the quotient of an étale groupoid equipped with an invariant Riemannian metric on the unit space. Such groupoids are called Riemannian étale groupoids and appear naturally in the study of Riemannian foliations.

A Riemannian foliation [14] is a foliation $(M, \mathcal{F})$ equipped with a bundle-like metric $\eta$. Such a bundle-like metric defines a holonomy invariant metric on the normal bundle of the foliation. Let $X$ be a complete transversal to the foliation $\mathcal{F}$ which means that $X$ is an immersed submanifold of $M$ which intersects every leaf of $\mathcal{F}$. The holonomy groupoid $\mathrm{G}$ associated to $X$ is an étale groupoid and the 
metric $\eta$ defines a $\mathrm{G}$-invariant metric on $X=\mathrm{G}_{0}$. In particular, if all the leaves of $\mathcal{F}$ are closed in $M$, then the groupoid $\mathrm{G}$ is proper and the quotient space $X / \mathrm{G}$ is an orbifold.

Let $\mathrm{G}$ be an étale groupoid with an invariant Riemannian metric $\eta$. We consider the convolution algebra $\mathcal{C}^{\infty} \rtimes \mathrm{G}$ of compactly supported smooth functions on $\mathrm{G}$. This algebra is well studied in noncommutative geometry. In particular, its cyclic homology was computed by Brylinsky and Nistor [3] and Crainic [7]. When there is a $\mathrm{G}$-invariant symplectic form on $\mathrm{G}_{0}$, deformation quantization of the groupoid algebra $\mathcal{C}^{\infty} \rtimes \mathrm{G}$ was constructed by the last author [22]. As a first step toward the algebraic index theory, we construct explicit cyclic (co)cycles on the deformation quantization $\mathcal{A}^{\hbar} \rtimes \mathrm{G}$ of $\mathcal{C}^{\infty} \rtimes \mathrm{G}$. More precisely, we construct a quasi-isomorphism $\Psi$ from the cyclic chain complex of the algebra $\mathcal{A}^{\hbar} \rtimes \mathrm{G}$ to the de Rham complex of compactly supported simplicial differential forms on the inertia groupoid $\Lambda G$ of $G$, and a quasi-isomorphism $\Phi$ from the de Rham complex on the inertia groupoid $\Lambda \mathrm{G}$ to the cyclic cochain complex of $\mathcal{A}^{\hbar} \rtimes \mathrm{G}$. These quasi-isomorphisms generalize our constructions in [18, Sec. 5]. The new input in this paper is that instead of working with the inertia orbifold which is the quotient space defined by the inertia groupoid, we construct $\Phi$ and $\Psi$ on the simplicial spaces associated to the nerves of the inertia groupoid $\Lambda \mathrm{G}$. This improvement allows dropping the assumption that the groupoid $G$ under consideration is proper, and also suggests a further generalization to étale groupoids without invariant Riemannian structures.

In algebraic index theory one wants to determine topological formulas for the Chern character of elements of the $K_{0}$-group of a deformation quantization. In the work [18], we obtained a complete answer to this question in the case of proper étale groupoids. To answer this question for general étale groupoids, we need more tools in Lie algebra cohomology and homological algebras. We plan to discuss this in the future. Instead, we study an interesting example about a constant Dirac structure on a 3D torus, whose idea goes back to joint work of the third author with Weinstein [23]. The transformation groupoid of the group $\mathbb{Z}$ acting on a $2 \mathrm{D}$ torus $X$ by irrational translations appears naturally in the description of the geometry of the characteristic foliation associated to such a Dirac structure. Furthermore, the Dirac structure defines a $\mathbb{Z}$ invariant symplectic form on the $2 \mathrm{D}$ torus $X$. We discuss the topological formulas of $K_{0}$ elements of the deformation quantization $\mathcal{A}_{X}^{\hbar} \rtimes \mathbb{Z}$. Our formulas for the Chern characters agree with Connes' computations in [6].

This paper is organized as follows. In Section 1, we discuss the construction of a deformation quantization $\mathcal{A}^{\hbar} \rtimes \mathrm{G}$ and prove an equivariant normal form theorem for a $\mathrm{G}$-invariant symplectic form on $\mathrm{G}_{0}$; in Section 2, we construct a quasi-isomorphism from the cyclic chain complex of $\mathcal{A}^{\hbar} \rtimes \mathrm{G}$ to the de Rham complex on the inertia groupoid $\Lambda \mathrm{G}$; and in Section 3, we propose a general index problem and discuss the examples of proper étale groupoids and constant Dirac structures on tori. 


\section{Quantized Convolution Algebras}

\subsection{DEFORMATION QUANTIZATION OVER ÉTALE GROUPOIDS}

Let $\mathrm{G}: \mathrm{G}_{1} \rightrightarrows \mathrm{G}_{0}$ be an étale Hausdorff Lie groupoid. Moreover, assume that $\mathrm{G}_{0}$ carries a $G$-invariant Riemannian metric $\eta$ and an invariant symplectic form $\omega$. Both tensor fields can be lifted to invariant forms on $G_{1}$ via pull-back by the source or target map. The resulting forms are again denoted by $\omega$ and $\eta$. Note that the Levi-Civita connection $\nabla^{\mathrm{LC}}$ corresponding to $\eta$ is $\mathrm{G}$-invariant, and that one can construct from it a torsion-free G-invariant symplectic connection $\nabla$ by putting

$$
\nabla_{Y} X:=\nabla_{Y}^{\mathrm{LC}} X+\Delta(X, Y), \quad X, Y \in \Gamma^{\infty}\left(T \mathrm{G}_{1}\right),
$$

where $\Delta \in \Gamma^{\infty}\left(T^{*} M \otimes T^{*} M \otimes T M\right)$ is the unique tensor field satisfying $\omega(-, \Delta)=$ $\Delta^{\prime}$ with the tensor field $\Delta^{\prime} \in \Gamma^{\infty}\left(T^{*} M \otimes T^{*} M \otimes T^{*} M\right)$ defined by

$$
\Delta^{\prime}(X, Y, Z):=\nabla_{Z}^{\mathrm{LC}} \omega(X, Y)+\nabla_{Y}^{\mathrm{LC}} \omega(X, Z), \quad X, Y, Z \in \Gamma^{\infty}\left(T \mathrm{G}_{1}\right) .
$$

One checks easily that $\nabla$ is a torsion-free symplectic connection on $G_{1}$ (cf. [8, Sec. 2.5]).

EXAMPLE 1.1. As pointed out in Section 1, the groupoid associated to a Riemannian foliation is an étale and Hausdorff Lie groupoid which is proper if and only if all leaves are closed. Other examples of étale groupoids are the transformation groupoid $\Gamma \ltimes M$ of a discrete group $\Gamma$ acting on a smooth manifold $M$ or the Haefliger groupoid of local diffeomorphisms on a manifold $M$. The cotangent bundle of a (Riemannian) étale groupoid even carries a natural invariant symplectic structure.

Next, consider the formal Weyl algebra bundle $\mathcal{W} \rightarrow \mathrm{G}_{0}$. Its fiber over a point $x \in \mathrm{G}_{0}$ is given by the formal Weyl algebra over $T_{x} \mathrm{G}_{0}$, which means the space of formal power series of the form

$$
a=\sum_{k \in \mathbb{N}, \alpha \in \mathbb{N}^{2 d}} \hbar^{k} a_{k, \alpha} y^{\alpha}, \quad a_{k, \alpha} \in \mathbb{C},
$$

where $2 d=\operatorname{dim} G$ and $y^{1}, \ldots, y^{2 d}$ denotes a symplectic basis of the tangent space $T_{x} \mathrm{G}_{0}$. The product on $T_{x} \mathrm{G}_{0}$ is given by

$$
a \circ b=\left.\left(\exp \left(-\frac{i \hbar}{2} \omega^{i j} \frac{\partial}{\partial y^{i}} \frac{\partial}{\partial z^{j}}\right) a(y, \hbar) b(z, \hbar)\right)\right|_{z=y} .
$$

Observe that $\mathcal{W}$ can be identified as the associated bundle $F \mathrm{G}_{0} \times_{\mathfrak{s p}_{d}} \mathbb{W}^{2 d}$, where $F \mathrm{G}_{0}$ denotes the symplectic frame bundle of $\mathrm{G}_{0}, \mathbb{R}^{2 d}$ carries its canonical symplectic structure, and $\mathbb{W}^{2 d}$ denotes the formal Weyl algebra on $\mathbb{R}^{2 d}$. By assigning degree 1 to every basis element $y_{i}$, and degree 2 to the formal parameter $\hbar$, 
the formal Weyl algebra bundle becomes a bundle of filtered algebras. Denote by $\mathcal{W}_{\geq p}$ the bundle of elements of degree $\geq p$ which means of all elements of form (1.1) where $a_{k, \alpha}=0$, if $2 k+|\alpha|<p$. Next, note that the symplectic connection $\nabla$ lifts to a connection on forms with values in the Weyl algebra bundle:

$$
\nabla: \Omega^{\bullet}\left(\mathrm{G}_{0}, \mathcal{W}\right) \rightarrow \Omega^{\bullet}\left(\mathrm{G}_{0}, \mathcal{W}\right)
$$

This connection in general has non-vanishing curvature.

According to Fedosov [8, Sec. 5.2] there exists a flat connection $D: \Omega^{\bullet}(M, \mathcal{W})$ $\rightarrow \Omega^{\bullet}\left(\mathrm{G}_{0}, \mathcal{W}\right)$ of the form

$$
D=\nabla+\frac{i}{\hbar}[A,-] \text {, }
$$

where $A \in \Omega^{1}\left(\mathrm{G}_{0}, \mathcal{W}_{\geq 3}\right)$. By construction, $A$ and $D$ are $\mathrm{G}$-invariant since $\omega$ and $\nabla$ are invariant. Since $D$ satisfies the Leibniz rule, the space of flat sections

$$
\mathcal{A}^{\hbar}\left(\mathrm{G}_{0}\right):=\left\{f \in \Gamma^{\infty}\left(\mathrm{G}_{0}, \mathcal{W}\right) \mid D f=0\right\}
$$

inherits an associative product $\star$ from $\mathcal{W}$. Moreover, the symbol map $\sigma: \mathcal{A}^{\hbar}\left(\mathrm{G}_{0}\right) \rightarrow$ $\mathcal{C}^{\infty}\left(\mathrm{G}_{0}\right)$ which locally is given by

$$
f=\sum_{k \in \mathbb{N}, \alpha \in \mathbb{N} 2 d} f_{k, \alpha} y^{\alpha} \hbar^{k} \mapsto f_{0}:=\sum_{k \in \mathbb{N}} f_{k, 0} \hbar^{k}, \quad f_{k, \alpha} \in \mathcal{C}^{\infty}\left(\mathrm{G}_{0}\right)
$$

is a linear isomorphism. This implies that $\mathcal{A}^{\hbar}\left(\mathrm{G}_{0}\right)$ is a deformation quantization over $\mathrm{G}_{0}$ which means that the following properties hold true:

(1) One has

$$
\mathfrak{q}\left(f_{1}\right) \star \mathfrak{q}\left(f_{2}\right)=\sum_{k \in \mathbb{N}} \mathfrak{q}\left(c_{k}\left(f_{1}, f_{2}\right)\right) \hbar^{k}
$$

for all $f_{1}, f_{2} \in \mathcal{C}^{\infty}\left(\mathrm{G}_{0}\right)$. Here, $\mathfrak{q}$ is the inverse of $\sigma$, and the $c_{k}$ are appropriate bidifferential operators on $\mathcal{C}^{\infty}\left(\mathrm{G}_{0}\right)$ such that $c_{0}$ is the commutative product of functions.

(2) $\mathfrak{q}(1)$ acts as unit element with respect to the product $\star$.

(3) One has

$$
\left[\mathfrak{q}\left(f_{1}\right), \mathfrak{q}\left(f_{2}\right)\right]_{\star}:=\mathfrak{q}\left(f_{1}\right) \star \mathfrak{q}\left(f_{2}\right)-\mathfrak{q}\left(f_{2}\right) \star \mathfrak{q}\left(f_{1}\right)=-i \hbar \mathfrak{q}\left(\left\{f_{1}, f_{2}\right\}\right)+o\left(\hbar^{2}\right)
$$

for all $f_{1}, f_{2} \in \mathcal{C}^{\infty}\left(\mathbf{G}_{1}\right)$.

Note that the bidifferential operators $c_{k}$ are uniquely determined by $D$, and that $\star$ is G-invariant by construction. Moreover, since $D$ is a differential operator, * is local and one even obtains a G-sheaf of deformed algebras $\left(\mathcal{A}^{\hbar}, \star\right)$. From this sheaf one can form the crossed product algebra $\mathcal{A}^{\hbar} \rtimes \mathrm{G}$. The underlying linear space is given by $\Gamma_{\mathrm{cpt}}^{\infty}\left(\mathrm{G}_{1}, s^{*} \mathcal{A}^{\hbar}\right)$, the space of all smooth functions $F: \mathrm{G}_{1} \rightarrow \mathcal{W}$ with compact support such that for every $g \in \mathrm{G}_{1}$ there is an open neighborhood $U$ 
of $g$ and an element $f \in \mathcal{A}^{\hbar}\left(\int(\mathcal{U})\right)$ such that $F_{\mid U}=f \circ s_{\mid U}$. The product on $\mathcal{A}^{\hbar} \rtimes \mathrm{G}$ is the convolution product given as follows:

$$
F_{1} \star_{\mathrm{c}} F_{2}(g):=\sum_{g_{1} g_{2}=g} F_{1}\left(g_{1}\right) g_{2} \star F_{2}\left(g_{2}\right), \quad F_{1}, F_{2} \in \Gamma_{\mathrm{cpt}}^{\infty}\left(\mathrm{G}_{1}, s^{*} \mathcal{A}^{\hbar}\right) .
$$

Note that here we have used that $\mathrm{G}$ acts from the right on the sheaf $\mathcal{A}^{\hbar}$. As pointed out in [22], the crossed product algebra $\mathcal{A}^{\hbar} \rtimes \mathrm{G}$ forms a deformation quantization of the convolution algebra $\mathcal{C}^{\infty} \rtimes \mathrm{G}$ along the noncommutative Poisson structure induced by $\omega$. Moreover, the invariant algebra $\left(\mathcal{A}^{\hbar}\right)^{\mathrm{G}}$ forms a deformation quantization of the algebra $\mathcal{C}^{\infty}\left(\mathrm{G}_{0}\right)^{\mathrm{G}}$ of invariant smooth functions on $\mathrm{G}_{0}$.

Our constructions in this paper rely crucially on the existence of an invariant Riemannian structure. For the case where this condition fails, a more general theory has been developed by Bressler et al. [1].

For the algebraic index theory over the étale groupoid $G$ one has to obtain a precise understanding of the cyclic cohomology theory of the deformed convolution algebra $\mathcal{A}^{\hbar} \rtimes \mathrm{G}$. It is given as follows, with proof provided in the following section:

$$
H C^{\bullet}\left(\mathcal{A}^{\hbar} \rtimes \mathrm{G}\right) \cong \bigoplus_{k \geq 0} H^{\bullet-2 k}(B \Lambda \mathrm{G}, \mathbb{C}((\hbar))) .
$$

This implies in particularly that the space of traces on $\mathcal{A}^{\hbar} \rtimes \mathrm{G}$ is isomorphic to $H^{0}(B \Lambda \mathrm{G}, \mathbb{C}((\hbar)))$. In Equation (1.6), $B \mathrm{H}$ denotes the classifying space of an étale groupoid $H$, and $\Lambda \mathrm{G}$ is the inertia groupoid of $\mathrm{G}$. The latter is defined as the transformation groupoid $B_{0} \rtimes \mathrm{G}$, where $B_{0}:=\left\{g \in \mathrm{G}_{1} \mid s(g)=t(g)\right\}$ is the space of loops of $G$ on which $G$ acts by conjugation

$$
\mathrm{G}_{1 s} \times_{\sigma_{0}} B_{0} \rightarrow B_{0}, \quad(h, g) \mapsto h g h^{-1},
$$

with $\sigma_{0}: B_{0} \rightarrow \mathrm{G}_{0}$ the moment map given by $\sigma_{0}(g)=s(g)$. Note that, although $B_{0}$ is in general disconnected, $\sigma_{0}$ is an immersion. Observe also that the inertia groupoid $\Lambda G$ is étale, respectively proper, when $G$ is.

Before we construct the explicit chain map realizing the isomorphism in Equation (1.6), let us explain in some more detail how to determine the cohomology of the classifying space of an étale groupoid and its inertia groupoid.

\subsection{COHOMOLOGY OF THE INERTIA GROUPOID}

For any étale Lie groupoid $\mathrm{G}$, let $\mathrm{G}_{k}$ be the space of composable $k$-tuples of arrows:

$$
\mathrm{G}_{k}=\left\{\left(g_{1}, \ldots, g_{k}\right) \in \mathrm{G}^{k} \mid s\left(g_{i}\right)=t\left(g_{i+1}\right), i=1, \ldots, k-1\right\} .
$$


These spaces form a simplicial manifold with face operators $\delta_{i}: \mathrm{G}_{k} \rightarrow \mathrm{G}_{k-1}, i=$ $1, \ldots, k$ given by

$$
\delta_{i}\left(g_{1}, \ldots, g_{k}\right)= \begin{cases}\left(g_{2}, \ldots, g_{k}\right), & \text { for } i=0, \\ \left(g_{1}, \ldots, g_{i} g_{i+1}, \ldots, g_{k}\right), & \text { for } 1 \leq i \leq k-1, \\ \left(g_{1}, \ldots, g_{k-1}\right), & \text { for } i=k .\end{cases}
$$

With this structure, one obtains the following differentials on the space of differential forms on $\mathbf{G}_{\bullet}$. First one has the exterior derivative $d: \Omega^{p}\left(\mathrm{G}_{q}\right) \rightarrow \Omega^{p+1}\left(\mathrm{G}_{q}\right)$. Second, the simplicial structure defines the differential $\delta: \Omega^{p}\left(\mathrm{G}_{q}\right) \rightarrow \Omega^{p}\left(\mathrm{G}_{q+1}\right)$ given by

$$
\delta(\alpha):=\sum_{i=0}^{q}(-1)^{i} \delta_{i}^{*}(\alpha) .
$$

Since $\delta$ is given by pull-backs, the two differentials commute: $[d, \delta]=0$. The total complex computes the cohomology of the classifying space $B \mathrm{G}$ :

$$
H^{\bullet}\left(\operatorname{Tot}\left(\Omega^{\bullet}\left(\mathrm{G}_{\bullet}\right), d+\delta\right) \cong H^{\bullet}(B \mathrm{G}, \mathbb{C}) .\right.
$$

Likewise, one can consider the compactly supported differential forms $\Omega_{\mathrm{cpt}}^{\bullet}\left(\mathrm{G}_{\bullet}\right)$. Then one has to push forward to define the derivative by

$$
\partial(\alpha)=\sum_{i=0}^{q}(-1)^{i}\left(\delta_{i}\right)_{*}(\alpha),
$$

which now lowers degrees: $\partial: \Omega_{\mathrm{cpt}}^{p}\left(\mathrm{G}_{q}\right) \rightarrow \Omega_{\mathrm{cpt}}^{p}\left(\mathrm{G}_{q-1}\right)$. One easily verifies that $\left(\operatorname{Tot}\left(\Omega_{\mathrm{cpt}}^{\bullet}\left(\mathrm{G}_{\bullet}\right), d, \partial\right)\right.$ forms a mixed complex.

We will apply this to the classifying space of the inertia groupoid $\Lambda \mathrm{G}=B_{0} \rtimes \mathrm{G}$. Rather than using the simplicial space associated to $\Lambda \mathrm{G}$, we will use the isomorphic simplicial space given by the higher Burghelea spaces:

$$
B_{k}:=\left\{\left(g_{0}, \ldots, g_{k}\right) \in \mathrm{G}_{k+1} \mid s\left(g_{k}\right)=t\left(g_{0}\right)\right\} .
$$

The face operators are simply given by

$$
\delta_{i}\left(g_{0}, \ldots, g_{k}\right)= \begin{cases}\left(g_{0}, \ldots, g_{i} g_{i+1}, \ldots, g_{k}\right), & \text { for } 0 \leq i \leq k-1, \\ \left(g_{k} g_{0}, \ldots, g_{k-1}\right), & \text { for } i=k .\end{cases}
$$

There is in fact a canonical cyclic structure on this space given by

$$
t_{k}\left(g_{0}, \ldots, g_{k}\right)=\left(g_{k}, g_{0}, \ldots, g_{k-1}\right) .
$$

We will use this cyclic structure in the following way: suppose that $\delta_{\bullet}$ is a cyclic object in the category $S h(G)$ of $G$-sheaves on $G_{0}$, for example the cyclic sheaf resulting from a sheaf of $\mathrm{G}$-algebras. This yields the cyclic vector space

$$
\Gamma_{\mathrm{cpt}}\left(B_{k}, \sigma_{k}^{-1} \mathcal{S}_{k}\right)
$$


where $\sigma_{k}: B_{k} \rightarrow G_{0}$ is the map defined by

$$
\sigma_{k}\left(g_{0}, \ldots, g_{k}\right)=s\left(g_{0}\right) \text {. }
$$

The cyclic structure is given by combining the cyclic structure on $B_{\bullet}$ together with the cyclic structure on $\mathcal{S}_{\bullet}$, where one twists the $k$ th face operator and the cyclic operator by the automorphism $\theta_{g_{0} \ldots g_{k}}$ at $\left(g_{0}, \ldots, g_{k}\right) \in B_{k}$. See [7] for explicit formulas.

\subsection{MIXED COMPLEXES AND S-MORPHISMS}

Finally in this section, let us briefly recall some concepts about mixed complexes as we will need these later.

Recall that by a mixed complex one understands a triple $\left(C_{\bullet}, b, B\right)$ where $C_{\bullet}$ is a graded object in some abelian category, $b: C_{k} \rightarrow C_{k-1}$ is a graded map of degree -1 and $B: C_{k} \rightarrow C_{k+1}$ a graded map of degree +1 such that the relations $b^{2}=B^{2}=$ $b B+B b=0$ are satisfied. A mixed complex gives rise to a first quadrant double complex $\mathcal{B C}$

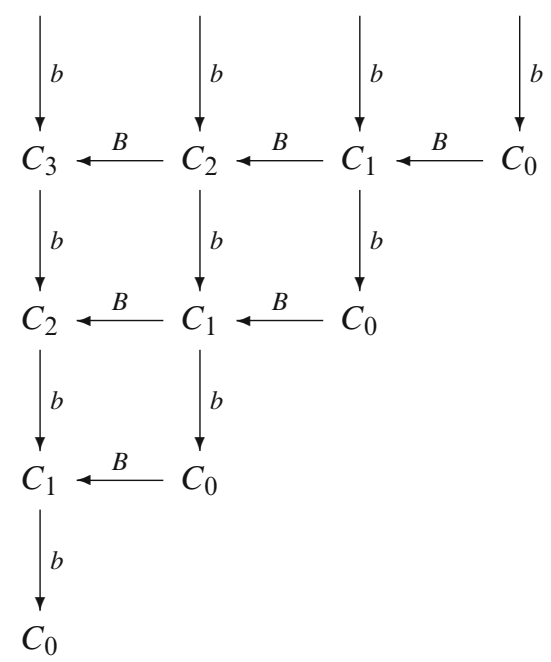

The Hochschild homology $H_{\bullet}(C)$ of a mixed complex $C=\left(C_{\bullet}, b, B\right)$ is defined as the homology of the $\left(C_{\bullet}, b\right)$-complex. The cyclic homology $H_{\mathbf{\bullet}}(C)$ is defined as the homology of the total complex associated to the double complex $\mathcal{B} C$. In this paper, $C_{\text {• }}$ will always be the Hochschild complex of a unital algebra, $b$ the Hochschild boundary, and $B$ Connes' coboundary.

Every mixed complex $\left(C_{\bullet}, b, B\right)$ comes with a natural periodicity morphism $S: \operatorname{Tot}_{\bullet} \mathcal{B C} \rightarrow \operatorname{Tot}_{\bullet} \mathcal{B C}$ of degree -2 which is given by the canonical projection $\operatorname{Tot}_{k} \mathcal{B} C \rightarrow \operatorname{Tot}_{k-2} \mathcal{B} C$. 
By an S-morphism between two mixed complexes $\left(C_{\bullet}, b_{C}, B_{C}\right)$ and $\left(D_{\bullet}, b_{D}, B_{D}\right)$ one now understands a morphism of complexes $f: \operatorname{Tot}_{\bullet} \mathcal{B} C \rightarrow \operatorname{Tot}_{\bullet} \mathcal{B} D$ which commutes with the corresponding periodicity maps $S_{C}$ and $S_{D}$ (cf. [13]). Note that such an S-morphism $f$ induces a morphism on the corresponding Hochschild complexes. The important observation which we will tacitly use throughout this paper is that $f$ is a quasi-isomorphism with respect to cyclic homology if and only if it induces an isomorphism on Hochschild homology (see [13, Prop. 2.5.15]).

\subsection{AN EQUIVARIANT NORMAL FORM THEOREM}

For later purposes in this article we derive an equivariant normal form theorem out of the following result.

THEOREM 1.2 (Moser Theorem - Equivariant Version). Let $\mathrm{G}$ be a Riemannian étale groupoid, and $\iota: M \hookrightarrow \mathrm{G}_{0}$ an invariant submanifold of the base. Let $\omega_{0}$ and $\omega_{1}$ be two $\mathbf{G}$-invariant symplectic forms on $\mathbf{G}_{0}$ such that $\omega_{0 x}=\omega_{1 x}$ for all $x \in M$. Then there exist $\mathrm{G}$-invariant open neighborhoods $U_{0}$ and $U_{1}$ of $M$ in $\mathrm{G}_{0}$ and a $\mathrm{G}$-equivariant $\varphi: U_{0} \rightarrow U_{1}$ such that $\iota=\varphi \circ \iota$ and $\varphi^{*} \omega_{1}=\omega_{0}$.

Proof. We only provide a sketch and extend the proof as presented in [4, Sec. 7.3] to the equivariant setting. To this end first note that the G-invariant Riemannian metric on $\mathrm{G}_{0}$ gives rise to an equivariant exponential map exp: $W \rightarrow$ $\mathrm{G}_{0}$ on a $\mathrm{G}_{0}$-invariant neighborhood $W$ of the zero-section of $T \mathrm{G}_{0}$. After restriction of $W$ we can assume that $\exp _{T_{x} \mathrm{G}_{0} \cap W}$ is injective for all $x \in \mathrm{G}_{0}$. Let $N \rightarrow M$ be the normal bundle of $M$ in $\mathrm{G}_{0}$. Obviously, $\mathrm{G}$ acts on $N$. The restriction $\psi:=$ $\exp _{\mid \tilde{W}}: \tilde{W} \rightarrow U_{0}$ with $\tilde{W}:=W \cap N$ and $U_{0}:=\exp (\tilde{W})$ is then a G-invariant tubular neighborhood of $M \hookrightarrow \mathrm{G}_{0}$. Observe that $H: U_{0} \times[0,1] \rightarrow U_{0},(x, t) \mapsto \psi^{-1}(t \psi(x))$ is then a G-equivariant homotopy which by the proof of Poincaré's lemma gives rise to a G-invariant 1-form $v$ on $U_{0}$ such that $\omega_{1}-\omega_{0}=d v$. After possibly shrinking $U_{0}$, the family of closed 2-forms $\omega_{t}=(1-t) \omega_{0}+t \omega_{1}=\omega_{0}+t d v$ is in fact a symplectic family for all $t \in[0,1]$. Now let $V_{t}$ be the unique vector field on $U_{0}$ such that $\omega_{t}\left(V_{t},-\right)=-v$. Integration of $V_{t}$ provides (after possibly shrinking $U_{0}$ further), a G-equivariant isotopy $\varrho: U_{0} \times[0,1] \rightarrow \mathrm{G}_{0}$ such that $\varrho_{t}^{*} \omega_{t}=\omega_{0}$ for all $t \in[0,1]$. Since $V_{t \mid M}=0$, one has $\varrho_{t \mid M}=\operatorname{id}_{M}$. By putting $\varphi:=\varrho_{1}$ and $U_{1}:=\varrho_{1}\left(U_{0}\right)$, we obtain the diffeomorphism with the claimed properties.

Now assume that $M \subset \mathrm{G}_{0}$ is an invariant submanifold and $\mathrm{G}_{0}$ carries an invariant symplectic form $\omega$ such that the restriction $\omega_{\mid M}$ is a symplectic form on $M$. Observe that by assumptions the invariant Riemannian and symplectic structures on $\mathrm{G}_{0}$ induce a $\mathrm{G}$-invariant almost complex structure on $\mathrm{G}_{0}$. This gives rise to a an invariant Hermitian structure on the normal bundle $\pi: N \rightarrow M$ to $T M$ in $\mathrm{G}_{0}$ which in particular means that $N$ is a complex vector bundle over $M$. 
From the Riemannian structure on $\mathrm{G}_{0}$ one can construct a $\mathrm{G}$-invariant Hermitian connection $\nabla^{N}$ on $N$. Choose local complex bundle coordinates $z$ on $N$, and put

$$
\omega_{N}:=d\left(z^{*} \nabla^{N} z\right)+\pi^{*} \omega_{\mid M} .
$$

Then $\omega_{N}$ is a G-invariant symplectic form on a neighborhood of the zero-section of $N$. The above equivariant Moser Theorem implies the following.

COROLLARY 1.3 (Equivariant Normal Form). There exists a G-equivariant diffeomorphism $\varphi: U \rightarrow V$ where $U, V$ are invariant sufficiently small open neighborhoods of $M$ in $\mathbf{G}$, respectively, the normal bundle $N$, such that $\varphi^{*} \omega_{N}=\omega_{\mid U}$.

\section{An Explicit Chain Map}

In this section, we present the computation of the cyclic homology and cohomology of the deformation $\mathcal{A}^{\hbar} \rtimes \mathrm{G}$ of the convolution algebra of $\mathrm{G}$ using an explicit chain map

$$
\operatorname{Tot}\left(\mathcal{B} C \cdot\left(\mathcal{A}^{\hbar} \rtimes \mathrm{G}\right)\right) \stackrel{\Psi}{\longrightarrow} \operatorname{Tot}\left(\Omega_{c}^{\bullet}\left(B_{\bullet}\right)\right),
$$

as well as its transpose

$$
\operatorname{Tot}\left(\Omega^{\bullet}\left(B_{\bullet}\right)\right) \stackrel{\Phi}{\longrightarrow} \operatorname{Tot}\left(\mathcal{B} C \cdot\left(\mathcal{A}^{\hbar} \rtimes \mathrm{G}\right)\right) .
$$

These maps will implement the isomorphisms in Equation (1.6).

\subsection{TWISTED CYCLIC DENSITIES}

Observe first that the above defined maps $\sigma_{p}: B_{p} \rightarrow \mathrm{G}_{0}$ are immersions which embed each connected component as a closed submanifold of $G_{0}$ because $G_{0}$ is equipped with an invariant Riemannian metric. For future use, we introduce the locally constant function $2 \ell: B_{p} \rightarrow \mathbb{N}$ given by the codimension of the above mentioned embedding.

The pull-back of the Weyl algebra bundle $\sigma_{p}^{*} \mathcal{W}$ is a bundle of unital associative algebras over $B_{p}$, which comes equipped with a canonical fiberwise family of automorphisms

$$
\theta_{g_{0} \ldots g_{p}} \in \operatorname{Aut}\left(\left(\sigma_{p}^{*} \mathcal{W}\right)_{\left(g_{0}, \ldots, g_{p}\right)}\right) .
$$

This can be seen as follows. The Weyl algebra bundle $\mathcal{W} \rightarrow \mathrm{G}_{0}$ forms a $\mathrm{G}$-vector bundle, so its fiber $\left(\sigma_{p}^{*} \mathcal{W}_{\mathrm{G}}\right)_{\left(g_{0}, \ldots, g_{p}\right)}=\mathcal{W}_{s\left(g_{0}\right)}$ will carry an action of the loop $g_{0} \cdots g_{p}$ for every $\left(g_{0}, \ldots, g_{p}\right) \in B_{p}$. Since $G$ is étale, this loop will act on the tangent space $T_{s\left(g_{0}\right)} \mathrm{G}_{0}$ and because the symplectic form $\omega$ is $\mathrm{G}$-invariant, this defines an element $g_{0} \cdots g_{p} \in \operatorname{Sp}\left(T_{s\left(g_{0}\right)} \mathrm{G}_{0}\right)$. In fact, this defines a section of the bundle of symplectic groups $\sigma_{p}^{*} \operatorname{Sp}\left(T \mathrm{G}_{0}\right) \rightarrow B_{p}$ associated to the symplectic vector bundle 
$\sigma_{p}^{*} T \mathrm{G}_{0}$ over $B_{p}$. Using the fact that the symplectic group of a symplectic vector space acts by automorphisms on its associated Weyl algebra, this defines the family of automorphisms $\theta$ above.

Using the invariant metric we obtain a decomposition

$$
T_{x} \mathrm{G}_{0}=T_{x} B_{p} \oplus T_{x} \sigma_{p},
$$

for all $x \in B_{p}$, where $T \sigma_{p}$ denotes the normal bundle to $\sigma_{p}: B_{p} \rightarrow \mathrm{G}_{0}$. Because of the G-invariance of the symplectic form $\omega$ on $\mathrm{G}_{0}$, this is a decomposition into symplectic subspaces. This direct sum decomposition factors the Weyl algebra as the tensor product $\mathcal{W}\left(T_{x} \mathrm{G}_{0}\right)=\mathcal{W}\left(T_{x} B_{p}\right) \otimes \mathcal{W}\left(T_{x} \sigma_{p}\right)$. Clearly the automorphism $\theta$ is the identity on the first factor.

Let

$$
\left(\tau_{0}^{\theta}, \tau_{2}^{\theta}, \ldots, \tau_{2 d-2 \ell}^{\theta}\right) \in \operatorname{Tot}^{2 d-2 \ell}(\mathcal{B} C(\mathcal{W}))
$$

be the twisted cyclic cocycle on the Weyl algebra $\left(\sigma_{p}^{*} \mathcal{W}\right)_{\left(g_{0}, \ldots, g_{p}\right)}=\mathcal{W}_{s\left(g_{0}\right)}$ as constructed in [18], with the twist given by the automorphism $\theta=\theta_{g_{0} \ldots g_{p}}$. It has the form

$$
\tau_{2 m}^{\theta}=\tau_{2 m} \# \operatorname{tr}_{\theta_{g}},
$$

where \# is the external product in cyclic cohomology with respect to the tensor product decomposition $\mathcal{W}\left(T_{x} \mathrm{G}_{0}\right)=\mathcal{W}\left(T_{x} B_{p}\right) \otimes \mathcal{W}\left(T_{x} \sigma_{p}\right)$ induced by (2.1). The cocycle $\tau_{2 m}$ is the untwisted cocycle of [18] on $\mathcal{W}\left(T_{s\left(g_{0}\right)} B_{p}\right)$ which extends the Hochschild cocycle of [10], and $\operatorname{tr}_{g_{g_{0} \ldots g_{p}}}$ is the twisted trace of [9] on $\mathcal{W}\left(T_{s\left(g_{0}\right)} \sigma_{p}\right)$. The definition of this trace uses an auxiliary invariant almost complex structure on $\mathrm{G}_{0}$ that we fix using the invariant Riemannian metric and symplectic form. This turns $T_{x} \sigma_{p}$ into a complex vector space and we have $\theta_{x} \in U\left(T_{x} \sigma_{p}\right)$.

Consider the sheaf $\sigma_{p}^{*} \mathcal{A}^{\hbar}$ of deformation quantizations over $B_{p}$. It has an acyclic resolution

$$
0 \longrightarrow \sigma_{p}^{*} \mathcal{A}^{\hbar} \longrightarrow \sigma_{p}^{*} \mathcal{W} \stackrel{D}{\longrightarrow} \Omega_{B_{p}}^{1} \otimes \sigma_{p}^{*} \mathcal{W} \stackrel{D}{\longrightarrow} \cdots
$$

with $D$ a Fedosov connection. The proof of this fact is the same as that of [19, Prop. 4.7.] given the equivariant normal form in Corollary 1.3. In a local trivialization of the Weyl algebra bundle induced by local Darboux coordinates, we can write $D=d+\hbar^{-1}[A,-]$, where $A \in \Omega^{1}\left(B_{p}\right) \otimes \sigma_{p}^{*} \mathcal{W}$ is invariant. Since two such trivializations differ by a symplectic transformation, $A$ is unique up to addition of a $\mathfrak{s p}\left(T \mathrm{G}_{0}\right)$-valued one-form.

Denote by $\mathcal{C}_{\bullet}\left(\sigma_{p}^{-1} \mathcal{A}^{\hbar}\right)=\sigma_{p}^{-1} \mathcal{C}_{\bullet}\left(\mathcal{A}^{\hbar}\right)$ the sheaf of Hochschild cochains on the sheaf of algebras $\sigma_{p}^{-1} \mathcal{A}^{\hbar}$ on $B_{p}$, equipped with the twisted Hochschild differential $b_{\theta}$. The associated cyclic bicomplex of sheaves has a total complex

$$
\text { BC. }\left(\sigma_{p}^{-1} \mathcal{A}^{\hbar}\right):=\bigoplus_{2 m \leq \bullet} \sigma_{p}^{-1} \mathcal{C}_{q-2 m}\left(\mathcal{A}^{\hbar}\right) \text {. }
$$


It is equipped with the twisted differential $b_{\theta}+B_{\theta}$. Let

$$
\psi_{2 m}^{i, p} \in \underline{\mathcal{H} o m}\left(\mathcal{C}_{2 m-i}\left(\sigma_{p}^{-1} \mathcal{A}^{\hbar}\right), \Omega_{B_{p}}^{i}\right),
$$

where $\underline{\mathcal{H} \text { om }}$ denotes the Hom-sheaf, be defined by

$$
\psi_{2 m}^{i, p}\left(a_{0} \otimes \cdots \otimes a_{2 m-i}\right):=\left(\frac{1}{\hbar}\right)^{i} \tau_{2 m}^{\theta}\left(\left(a_{0} \otimes \cdots \otimes a_{2 m-i}\right) \times\left(\sigma_{p}^{*} A\right)_{i}\right),
$$

where $\times$ means the shuffle product on Hochschild chains as in [18, Sec. 3.2].

PROPOSITION 2.1. The $\psi_{2 m}^{i, p}$ satisfy

$$
\begin{aligned}
(-1)^{i} d \psi_{2 m}^{i, p} & =\psi_{2 m}^{i, p} \circ b_{\theta}+\psi_{2 m+2}^{i, p} \circ B_{\theta}, \\
\delta_{i}^{*} \psi_{2 m}^{i, p-1} & =\psi_{2 m}^{i, p}, \quad \text { for all } 0 \leq i \leq p .
\end{aligned}
$$

Proof. First remark that the map $\sigma_{p}: B_{p} \rightarrow \mathrm{G}_{0}$ factors as

$$
\sigma_{p}=\sigma_{0} \circ \lambda_{p},
$$

where $\lambda_{p}: B_{p} \rightarrow B_{0}$ is given by $\lambda_{p}\left(g_{0}, \ldots, g_{p}\right)=g_{0} \ldots g_{p}$. While $\sigma_{0}$ is an immersion, $\lambda_{p}$ is étale as it is nothing but the $p$-fold composition of the face maps $\delta_{0}$. For $p=0$, the first equation reads

$$
(-1)^{i} d \circ \psi_{2 m}^{i, 0}=\psi_{2 m}^{i, 0} \circ b_{\theta}+\psi_{2 m+2}^{i, 0} B_{\theta},
$$

and was proved in [18, Prop. 5.5]. Using the factorization of $\sigma_{p}$ above and the fact that $\lambda_{p}$ is étale, the general case follows immediately.

As for the second equation, it should be interpreted as follows: obviously, the sheaf of differential forms is simplicial, i.e., there are canonical isomorphisms $\delta_{i}^{*} \Omega_{B_{p-1}}^{q} \cong \Omega_{B_{p}}^{q}$ for all $0 \leq i \leq p$. The same holds true for $\sigma_{p}^{-1} \mathcal{A}^{\hbar}$, where one has to be careful with the last one, i.e., $\delta_{p}$ as it involves the action of $\mathrm{G}$ on $\mathcal{A}^{\hbar}$. With this, the sheaf $\mathcal{C}_{2 m-i}\left(\sigma_{\bullet}^{-1} \mathcal{A}^{\hbar}\right)$, and therefore the Hom-sheaf in (2.2) is simplicial. Property $i$ ) now says that the $\psi_{2 m}^{i, \bullet}$ are compatible with all these isomorphisms of sheaves obtained by pull-back along the face maps. But this is easy to verify, given the factorization of $\sigma_{p}$ and the fact that $\lambda_{p}$ is compatible with the structure maps. In particular, for $\delta_{p}$ we use that the connection $A$ is G-invariant.

On the total sheaf of cyclic chains $\mathcal{B C}\left(\sigma_{p}^{-1} \mathcal{A}^{\hbar}\right)$, this proposition immediately implies:

COROLLARY 2.2. The morphism

$$
\psi^{p}:=\sum_{m=0}^{2 d-2 \ell} \psi_{2 d-2 \ell-2 m}^{2 d-2 \ell-\bullet}:\left(\sigma_{p}^{-1} \mathcal{B C} \bullet\left(\mathcal{A}^{\hbar}\right), b_{\theta}, B_{\theta}\right) \rightarrow\left(\Omega_{B_{p}}^{2 d-2 \ell-\bullet}, d, 0\right)
$$

is an S-morphism of mixed complexes of sheaves. 
Recall that $\ell$ is the locally constant function on $B_{p}$ measuring the codimension of the embeddings of the connected components of $B_{p}$ in G. The formula in Corollary 2.2 is therefore to be understood as a separate one on each of the connected components of $B_{p}$. With this, $2 d-2 \ell$ is by definition the dimension of the connected component of $B_{p}$ under consideration. Remark that with the grading as on the right-hand side, the de Rham differential lowers degree, so that the right-hand side indeed forms a mixed complex. The degree zero part of the $S$-morphism of complexes of sheaves is given by

$$
\left(\psi^{p}\right)_{0}=\psi_{2 d-2 \ell}^{2 d-2 \ell-\bullet, p}:\left(\mathrm{C}_{\bullet}\left(\sigma_{p}^{-1} \mathcal{A}^{\hbar}\right), b_{\theta}\right) \rightarrow\left(\Omega_{B_{p}}^{2 d-2 \ell-\bullet}, d\right),
$$

explicitly given by formula (2.3). Since $B_{p}$ is symplectic, we can consider Darboux coordinates $\left(x_{1}, \ldots, x_{2 d-2 \ell}\right)$ in a local chart. In such coordinates, the Fedosov connection is gauge equivalent to

$$
\sigma_{p}^{*} A=\sum_{i, j=0}^{2 d-2 \ell} \sigma_{p}^{*} \omega_{i j} y_{i} d x_{j},
$$

with $y_{i}$ the element in the Weyl algebra corresponding to $x_{i}$. Using this in (2.3) together with the explicit formula for $\tau_{2 d-2 \ell}$ in [18, Sec. 2], one finds

$$
\psi_{2 d-2 \ell}^{2 d-2 \ell-i, p}\left(a_{0} \otimes \cdots \otimes a_{i}\right)=*\left(a_{0} d a_{1} \wedge \ldots \wedge d a_{i}\right) \bmod \hbar,
$$

where $*$ denotes the symplectic Hodge star operator, $*: \Omega_{B_{p}}^{\bullet} \rightarrow \Omega_{B_{p}}^{2 d-2 \ell-\bullet}$, on $B_{p}$ as introduced in [2]. It is known that the quasi-classical limit of the Hochschild differential is given by

$$
\begin{aligned}
b\left(a_{0} \otimes \cdots \otimes a_{k}\right)= & \sum_{i=0}^{k-1}(-1)^{i} a_{0} \otimes \cdots \otimes\left\{a_{i}, a_{i+1}\right\} \otimes \cdots \otimes a_{k}+ \\
& +(-1)^{k}\left\{\theta\left(a_{k}\right), a_{0}\right\} \otimes a_{1} \otimes \cdots \otimes a_{k-1} \quad \bmod \hbar,
\end{aligned}
$$

which indeed is a complex by the fact that $\theta$ acts by Poisson automorphisms, because the symplectic form on $\mathrm{G}_{0}$ is $\mathrm{G}$-invariant. This is the twisted Poisson homology complex on $B_{k}$ of the sheaf $\sigma_{p}^{-1} \mathcal{C}_{\mathrm{G}_{0}}^{\infty}$ as in [16, Sec. 4]. The well-known morphism $\sigma_{p}^{-1} \mathcal{C}_{\bullet}\left(\mathcal{C}_{\mathrm{G}_{0}}^{\infty}\right) \rightarrow \sigma_{p}^{-1} \Omega_{\mathrm{G}_{0}}^{\bullet}$ given by

$$
a_{0} \otimes \cdots \otimes a_{k} \mapsto a_{0} d a_{1} \wedge \cdots \wedge d a_{k}
$$

defines a quasi-isomorphism to the more familiar Poisson complex on differential forms, however with the differential similarly twisted by $\theta$ as in $[3,7]$. Applying the equivariant normal form theorem Corollary 1.3, one can prove in the same way as Fedosov [9, Sec. 5] that the sheaf $\sigma_{p}^{-1} \mathcal{A}_{\mathrm{G}_{0}}^{\hbar}$ can be identified as the sheaf of deformation quantization of Weyl algebra valued functions on $B_{p}$. With such an observation, one argues in the same way as in [16, Prop. 5.4.] that there exists 
an inclusion $\phi_{0}: \mathcal{C}_{B_{p}}^{\infty} \hookrightarrow \sigma_{p}^{-1} \mathcal{C}_{\mathrm{G}_{0}}^{\infty}$ of sheaves of Poisson algebras which induces a quasi-isomorphism on the associated Poisson homology complexes and extends to a sheaf morphism $\phi: \mathcal{A}_{B_{p}}^{\hbar} \hookrightarrow \sigma_{p}^{-1} \mathcal{A}_{\mathrm{G}_{0}}^{\hbar}$ where $\mathcal{A}_{B_{p}}^{\hbar}$ is an appropriate deformation quantization of the symplectic manifold $\left(B_{p}, \sigma_{p}^{*} \omega\right)$. Because in the semiclassical limit $\phi_{0}$ induces an isomorphism on Poisson homology, $\phi$ is a quasi-isomorphism on the level of Hochschild chains. We now precompose $\psi_{2 d-2 \ell}^{2 d-2 \ell-i, p}$ with $\phi$. Since the $*$-operator maps the Poisson homology differential to the de Rham differential, one easily observes that in the quasi-classical limit of $\phi \circ \psi_{2 d-2 \ell}^{2 d-2 \ell-i, p}$ the twisting in the normal directions drops out, and reduces to (2.4) composed with * on the symplectic manifold $B_{p}$. But in this "untwisted" case, this is known to be a quasi-isomorphism, cf. [2], by the usual spectral sequence argument. Finally it follows that the morphism $\psi_{2 d-2 \ell}^{2 d-2 \ell-i, p}$ on the Hochschild complex is a quasiisomorphism. Since this is just the degree zero part of a full $S$-morphism $\psi^{p}$, the $S B I$ exact sequence in cyclic homology implies:

PROPOSITION 2.3. The S-morphism $\psi^{p}$ is a quasi-isomorphism.

\subsection{THE CHAIN MAP}

With the simplicial morphism $\psi^{\bullet}$ of mixed complexes of sheaves on $B_{\bullet}$, we can now prove our main theorem on the cyclic theory of the deformed convolution algebra $\mathcal{A}^{\hbar} \rtimes \mathrm{G}$ of the étale groupoid $\mathrm{G}$ :

\section{THEOREM 2.4. There exists a natural S-morphism}

$$
\Psi:\left(\operatorname{Tot}_{\bullet}\left(\mathcal{B} C \bullet\left(\mathcal{A}^{\hbar} \rtimes \mathrm{G}\right)\right), b, B\right) \rightarrow\left(\operatorname{Tot}_{\bullet}\left(\Omega_{c}^{2 d-2 \ell-\bullet}\left(B_{\bullet}, \mathbb{C}((\hbar))\right)\right), d+\partial, 0\right)
$$

which induces isomorphisms

$$
\begin{aligned}
& H H_{\bullet}\left(\mathcal{A}^{\hbar} \rtimes \mathrm{G}\right) \cong H_{\mathrm{cpt}}^{2 d-2 \ell-\bullet}(\Lambda \mathrm{G}, \mathbb{C}((\hbar))), \\
& H C \cdot\left(\mathcal{A}^{\hbar} \rtimes \mathrm{G}\right) \cong \bigoplus_{k \geq 0} H_{\mathrm{cpt}}^{2 d-2 \ell+2 k-\bullet}(\Lambda \mathrm{G}, \mathbb{C}((\hbar))) .
\end{aligned}
$$

Proof. The morphism $\Psi$ is the composition of the following three maps:

i) Restriction to loops: Since we have $C_{k}\left(\mathcal{A}^{\hbar} \rtimes \mathrm{G}\right) \cong \mathcal{A}_{\text {cpt }}^{\hbar}\left(\mathrm{G}^{\times(k+1)}\right)$, restriction to $B_{k} \subset \mathrm{G}^{\times(k+1)}$ induces a map

$$
C_{k}\left(\mathcal{A}^{\hbar} \rtimes \mathrm{G}\right) \rightarrow \Gamma_{\text {cpt }}\left(B_{k}, \mathcal{C}_{k}\left(\sigma_{k}^{-1} \mathcal{A}^{\hbar}\right)\right) .
$$

As in $[3,7]$ this map constitutes a morphism of cyclic objects where the righthand side carries the cyclic structure defined by that of $B$. combined with the twisted cyclic structure on $\sigma_{\bullet}^{-1} \mathcal{C}_{\bullet}\left(\mathcal{A}^{\hbar}\right)$. More explicitly, this means that the 
Hochschild differential

$$
b: \Gamma_{\mathrm{cpt}}\left(B_{k}, \mathcal{C}_{k}\left(\sigma_{k}^{-1} \mathcal{A}^{\hbar}\right)\right) \rightarrow \Gamma_{\mathrm{cpt}}\left(B_{k-1}, \mathcal{C}_{k-1}\left(\sigma_{k-1}^{-1} \mathcal{A}^{\hbar}\right)\right)
$$

is given by applying $\delta_{*}$ on the domain of sections combined with the stalkwise differential $b_{\theta}$ on the image.

ii) The cyclic Alexander-Whitney map: consider the bigraded vector space

$$
\left(\Gamma_{\mathrm{cpt}}\left(B_{p}, \mathrm{C}_{q}\left(\sigma_{p}^{-1} \mathcal{A}^{\hbar}\right)\right)\right)_{p, q \geq 0} .
$$

It is a cylindrical vector space, c.f. [11], where the simplicial and cyclic operators in the $p$-direction are given by those of the cyclic manifold $B_{\bullet}$, whereas in the $q$-direction they are given by the $\theta$-twisted cyclic structure associated to the stalks of the sheaf of algebras $\sigma_{p}^{-1} \mathcal{A}_{\mathrm{G}}$. The Eilenberg-Zilber theorem states that as $\left(\Gamma_{\text {cpt }}\left(B_{p}, \mathcal{C}_{q}\left(\sigma_{p}^{-1} \mathcal{A}^{\hbar}\right)\right)\right)_{p, q \geq 0}$ is a bisimplicial space, the Alexander-Whitney map

$$
\Gamma_{\mathrm{cpt}}\left(B_{k}, \mathrm{C}_{k}\left(\sigma_{k}^{-1} \mathcal{A}^{\hbar}\right)\right) \rightarrow \bigoplus_{p+q=k} \Gamma_{\mathrm{cpt}}\left(B_{p}, \mathrm{C}_{q}\left(\sigma_{p}^{-1} \mathcal{A}^{\hbar}\right)\right)
$$

is a quasi-isomorphism from the diagonal to the total simplicial space. Unfortunately, it is not a morphism of cyclic modules, as one can easily check from the explicit formulas [24, Sec. 8.5]. However, the Alexander-Whitney map is the degree zero part of an $S$-morphism which therefore is a quasi-isomorphism as well. In fact, applying the homological perturbation lemma, one can get explicit formulas for this map [12].

iii) The map $\psi$ in (2.2). By the first equation in Proposition 2.1, the morphism of sheaves

$$
\sum_{2 m \leq q} \psi_{2 q-2 m}^{q, p}: \bigoplus_{2 m \leq q} \sigma_{p}^{-1} \mathcal{C}_{q-2 m}\left(\mathcal{A}^{\hbar}\right) \rightarrow \Omega_{B_{p}}^{q}
$$

is an S-morphism of mixed complexes of sheaves, intertwining the differential $b_{\theta}+B_{\theta}$ with $d$. By the second equation in Proposition 2.1, it is simplicial over $B_{\bullet}$.

Combining these three maps, one finds the morphism $\Psi$. Since each of its three constituent maps are quasi-isomorphisms as explained above, the theorem follows.

As any $S$-morphism, the chain morphism above can be decomposed according to degree. Since in ii) above, the degree zero part is exactly the Alexander-Whitney map, whose explicit form is given as follows. 
COROLLARY 2.5. On the level of Hochschild homology, the isomorphism above is induced by the chain map

$$
\Psi_{0}: C \bullet\left(\mathcal{A}^{\hbar} \rtimes \mathrm{G}\right) \rightarrow \bigoplus_{p+q=\bullet} \Omega^{2 d-2 \ell-p}\left(B_{q}, \mathbb{C}((\hbar))\right)
$$

given by

$$
\Psi_{0}\left(a_{0} \otimes \cdots \otimes a_{k}\right)=\sum_{p+q=k} \psi_{2 d-2 \ell}^{2 d-2 \ell-p, q}\left(\left.a_{0} \star \cdots \star a_{q} \otimes a_{q+1} \otimes \cdots \otimes a_{k}\right|_{B_{q}}\right) .
$$

In the formula above, the products are given by the pointwise, noncommutative multiplication $\star$ in $\mathcal{A}^{\hbar}$.

\subsection{THE DUAL MAP}

The dual map

$$
\Phi:\left(\operatorname{Tot}^{\bullet}\left(\Omega^{\bullet}\left(B_{\bullet}\right), d+\delta, 0\right) \rightarrow\left(\operatorname{Tot}^{\bullet}\left(\mathcal{B} C \bullet\left(\mathcal{A}^{\hbar} \rtimes \mathrm{G}\right)\right), b, B\right)\right.
$$

follows by integration: for $\alpha \in \Omega^{p}\left(B_{q}\right)$ we have

$$
\Phi(\alpha)\left(a_{0} \otimes \cdots \otimes a_{p+q}\right):=\int_{B_{q}} \alpha \wedge \Psi^{p, q}\left(a_{0} \otimes \cdots \otimes a_{p+q}\right),
$$

where $\Psi^{p, q}: C_{p+q}\left(\mathcal{A}^{\hbar} \rtimes \mathrm{G}\right) \rightarrow \Omega_{c}^{2 d-2 \ell-p}\left(B_{q}, \mathbb{C}((\hbar))\right)$ are the components of the chain morphism $\Psi$ of Theorem 2.4.

It is easily verified that $\Phi$ is a chain morphism: let $\alpha=\left(\alpha_{p, q}\right)_{p+q=k}$ be a simplicial differential form on $B_{\bullet}$ of degree $k$, with $\alpha_{p, q} \in \Omega^{p}\left(B_{q}\right)$. Then we have

$$
\begin{aligned}
\Phi((d+\delta) \alpha)(a) & =\sum_{p+q=k} \int_{B_{q}} d \alpha_{p, q} \wedge \Psi^{p-1, q}(a)+\int_{B_{q+1}} \delta \alpha_{p, q} \wedge \Psi^{p, q}(a)= \\
& =\sum_{p+q=k}-\int_{B_{q}} \alpha_{p, q} \wedge d \Psi^{p-1, q}(a)+\int_{B_{q}} \alpha_{p, q} \wedge \partial \Psi^{p, q}(a)= \\
& =\sum_{p+q=k} \int_{B_{q}} \alpha_{p, q} \wedge \Psi^{p, q}((b+B)(a))= \\
& =((b+B) \Phi(\alpha))(a),
\end{aligned}
$$

where $a \in \operatorname{Tot}_{k}\left(\mathcal{B} C_{\bullet}\left(\mathcal{A}^{\hbar} \rtimes \mathrm{G}\right)\right)$. It follows from Theorem 2.4 that $\Phi$ is an $S$-morphism, i.e., compatible with the $S$-maps, and induces isomorphisms

$$
\begin{aligned}
& H H^{\bullet}\left(\mathcal{A}^{\hbar} \rtimes \mathrm{G}\right) \cong H^{\bullet}(\Lambda \mathrm{G}, \mathbb{C}((\hbar))), \\
& H C^{\bullet}\left(\mathcal{A}^{\hbar} \rtimes \mathrm{G}\right) \cong \bigoplus_{k \geq 0} H^{\bullet-2 k}(\Lambda \mathrm{G}, \mathbb{C}((\hbar))) .
\end{aligned}
$$




\section{An Index Problem and Examples}

In this section, we discuss the applications of the quasi-isomorphism $\Phi$ constructed in the previous section to an algebraic index problem. In Section 3.1, we will briefly recall our general set-up of algebraic index theory; in 3.2, we will explain an index theorem in the case where the Riemannian étale groupoid is proper; in Section 3.3, we will discuss an index theorem for quantization of a constant Dirac structure on a $3 \mathrm{D}$ torus.

\subsection{ALGEBRAIC INDEX PAIRING}

We recall the general theory of the noncommutative Chern character. Let $A$ be a unital algebra, and $M_{n}(A)$ be the algebra of $n \times n$ matrices with coefficients in $A$. Idempotent elements in $M_{n}(A)$ for $n \in \mathbb{N}$ modulo equivalence relations comprise the group $K_{0}(A)$. Let $I$ be the identity matrix in $M_{n}(A)$. The $k$-th Chern character of a $K_{0}$ element of $A$ represented by the idempotent $e \in M_{n}(A)$ is defined as follows,

$$
\begin{aligned}
C h_{k}(e) & :=\left(c_{k}, \ldots, c_{0}\right) \in \mathcal{B} C_{2 k}(A) \\
c_{i} & :=(-1)^{i} \frac{(2 i) !}{i !} \sum_{s_{0}, \ldots, s_{2 i}}\left(e-\frac{1}{2} I\right)_{s_{0} s_{1}} \otimes e_{s_{1} s_{2}} \otimes \ldots e_{s_{2 i} s_{0}} \in A \otimes \bar{A}^{\otimes 2 i}, \quad i \geq 1 \\
c_{0} & :=\sum_{s_{0}} e_{s_{0} s_{0}} \in A .
\end{aligned}
$$

It can be easily checked that $C h_{k}(e)$ is a cyclic cycle in the normalized $(b, B)$ bicomplex $\mathcal{B C}(A)$, and furthermore that equivalent idempotents in $K_{0}(A)$ give rise to the same homology class in the $2 k$-th cyclic homology group $H_{2 k}(A)$. Therefore, $C h_{k}$ defines a map from $K_{0}(A)$ to $H C_{2 k}(A)$.

By definition, there is a natural pairing between the cyclic homology and cohomology groups of an algebra $A$. Given a degree $2 k$ cyclic cocycle $\phi$ of $A$, the index pairing between a $K_{0}$-group element $e$ and the cyclic cocycle $\phi$ is defined to be

$$
\langle e, \phi\rangle:=\left\langle C h_{k}(e), \phi\right\rangle .
$$

We remark that if $A$ is not unital, then we need to adjoin a unit to $A$ in order to use the formula (3.1) to define the Chern character $C h_{k}$. For an element $e$ in $K_{0}(A), C h_{k}(e)$ is again a well defined class in $H_{2 k}(A)$, and the index pairing defined by equation (3.4) naturally extends.

Applying this index pairing (3.4) to the algebra $\mathcal{A}^{\hbar} \rtimes \mathrm{G}$, we come up with the following natural question.

QUESTION 3.1. Let $\mathrm{G}$ be a Hausdorff étale groupoid equipped with an invariant Riemannian metric on $\mathbf{G}_{0}$. Assume that there is a $\mathrm{G}$ invariant symplectic form $\omega$ on $\mathrm{G}_{0}$. We consider the quantization $\mathcal{A}^{\hbar} \rtimes \mathrm{G}$ as constructed in Section 1, and the $S$-morphism $\Psi:\left(\operatorname{Tot}_{\bullet}\left(\mathcal{B} C\left(\mathcal{A}^{\hbar} \rtimes \mathrm{G}\right)\right), b, B\right) \rightarrow\left(\operatorname{Tot}_{\bullet}\left(\Omega_{c}^{2 d-2 \ell-\bullet}\left(B_{\bullet}, \mathbb{C}((\hbar))\right)\right), d+\partial, 0\right)$ 
defined in Theorem 2.4. Let $e$ be an element in $K_{0}\left(\mathcal{A}^{\hbar} \rtimes \mathrm{G}\right)$. What is the cohomological formula for the class $\Psi\left(C h_{k}(e)\right)$ ?

We need to develop some Lie algebra cohomology tools as in [17, Sec. 5] in order to obtain a full answer to the above question. We plan to address this issue in future publications. In the following two subsections, we will discuss the answer to this question in two special cases.

\subsection{AN INDEX THEOREM FOR ORBIFOLDS}

In this subsection, we assume the groupoid $\mathrm{G}$ to be proper étale. This implies that the quotient space $X=\mathrm{G}_{0} / \mathrm{G}$ is an orbifold. In this case, the cyclic homology and cohomology of the algebra $\mathcal{A}^{\hbar} \rtimes \mathrm{G}$ were computed in full generality in [16, Sec. 5], and the index pairing (3.4) on the algebra $\mathcal{A}^{\hbar} \rtimes \mathrm{G}$ was computed in [18, Sec. 5]. We explain the results of [18] briefly.

We start with the observation that the $K_{0}$-group of an algebra is invariant under deformations. Therefore, the group $K_{0}\left(\mathcal{A}^{\hbar} \rtimes \mathrm{G}\right)$ is isomorphic to $K_{0}\left(\mathcal{C}^{\infty} \rtimes \mathrm{G}\right)$. According to [20], the $K_{0}$-group of the $C^{*}$-algebra completion of $\mathcal{C}^{\infty} \rtimes \mathrm{G}$ is computed by the topological $K_{0}$-group of $\mathrm{G}$-equivariant vector bundles on $\mathrm{G}_{0}$ since $\mathrm{G}$ is a proper étale groupoid. This group is also called the orbifold $K$-theory group of the underlying orbifold $X$. An element of the topological $K_{0}$-group of G-equivariant vector bundles consists of a pair of G-equivariant vector bundles $E$ and $F$ on $\mathrm{G}_{0}$, which are isomorphic outside a compact subset. Given such a pair of equivariant bundles $(E, F)$, we define an element $P(E)-P(F)$ in the group $K_{0}\left(\mathcal{C}^{\infty} \rtimes \mathrm{G}\right)$, where $P(E)$ and $P(F)$ are idempotents in $\mathcal{C}^{\infty,+} \rtimes \mathrm{G}$ and $P(E)-P(F)$ is compactly supported. (We have used the notation $\mathcal{C}^{\infty,+} \rtimes \mathrm{G}$ for the groupoid convolution algebra with a unit adjoined.) We refer to [17, Sec. 1] for the details of this construction. Furthermore, using the standard trick in [8, Sec. 6.1], we obtain an element $\widehat{P(E)}-\widehat{P(F)}$ in $K_{0}\left(\mathcal{A}^{\hbar} \rtimes \mathrm{G}\right)$ with the similar properties.

To compute the index pairing (3.4), we look at the cyclic cohomology of $\mathcal{A}^{\hbar} \rtimes \mathrm{G}$. As is explained in Section 2.3, the map

$$
\Phi: \operatorname{Tot}^{\bullet}\left(\Omega^{\bullet}\left(B_{\bullet}\right)\right) \rightarrow \operatorname{Tot}^{\bullet}\left(\mathcal{B} C\left(\mathcal{A}^{\hbar} \rtimes \mathrm{G}\right)\right)
$$

is a quasi-isomorphism. Therefore, the cyclic cohomology of $\mathcal{A}^{\hbar} \rtimes \mathrm{G}$ is isomorphic to the cohomology of $\operatorname{Tot}^{\bullet}\left(\Omega^{\bullet}\left(B_{\bullet}\right)\right)$. As $G$ is proper, the groupoid sheaf cohomology of $G$ is zero except in degree 0 . And the degree 0 cohomology of $G$ is equal to the space of invariant sections. This shows that the cohomology of $\operatorname{Tot}^{\bullet}\left(\Omega^{\bullet}\left(B_{\bullet}\right)\right)$ is equal to the cohomology of $\operatorname{Tot}^{\bullet}\left(\Omega^{\bullet}\left(B_{0}\right)^{\mathrm{G}}\right)=\operatorname{Tot}^{\bullet}\left(\Omega^{\bullet}\left(B_{0} / \mathrm{G}\right)\right)$, which is equal to the cohomology of the quotient $B_{0} / \mathrm{G}$. The space $B_{0} / \mathrm{G}$ is usually called the inertia orbifold associated to the orbifold $X=\mathrm{G}_{0} / \mathrm{G}$, and denoted by $\tilde{X}$.

In order to state our theorem, we introduce characteristic classes for a G-equivariant vector bundle $V$ on $B_{0}$. Let $R_{V}$ be the curvature form of a connection on 
the bundle $V$ on $B_{0}$. Define $C h_{\theta}(V) \in H^{\bullet}(\widetilde{X})$ by

$$
C h_{\theta}(V):=\operatorname{tr}\left(\theta \exp \left(\frac{R_{V}}{2 \pi \sqrt{-1}}\right)\right) .
$$

We consider the normal bundle $N$ of $B_{0}$ to be embedded as a submanifold of $\mathrm{G}$. It is easy to check that $N$ is $\mathrm{G}$ equivariant. Define $C h_{\theta}\left(\lambda_{-1} N\right) \in H^{\bullet}(\widetilde{X})$ by

$$
C h_{\theta}\left(\lambda_{-1} N\right):=\sum(-1)^{\bullet} C h_{\theta}\left(\wedge^{\bullet} N\right) .
$$

We proved the following theorem in [18].

THEOREM 3.2. Let $\mathrm{G}$ be a proper étale Lie groupoid. Let $E$ and $F$ be a pair of $\mathrm{G}$-equivariant vector bundles on $\mathrm{G}_{0}$ defining a $K_{0}$-group element $\widehat{P(E)}-\widehat{P(E)}$ in $K_{0}\left(\mathcal{A}^{\hbar} \rtimes \mathrm{G}\right)$, and let $\alpha=\left(\alpha_{2 k}, \ldots, \alpha_{0}\right) \in \operatorname{Tot}^{2 k} \Omega^{\bullet}(\widetilde{X})((\hbar))$ be a sequence of closed forms. Then, we have

$$
\begin{aligned}
& \left.\left\langle\Phi(\alpha), C h_{k}(\widehat{P(E)}-\widehat{P(F})\right)\right\rangle= \\
& \quad=\sum_{j=0}^{k} \int_{\widetilde{X}} \frac{1}{(2 \pi \sqrt{-1})^{j} m} \frac{\alpha_{2 j} \wedge \hat{A}\left(B_{0} / \mathrm{G}\right) \mathrm{Ch}_{\theta}\left(\iota^{*} E-\iota^{*} F\right) \exp \left(-\frac{\iota^{*} \Omega}{2 \pi \sqrt{-1} \hbar}\right)}{\mathrm{Ch}_{\theta}\left(\lambda_{-1} N\right)},
\end{aligned}
$$

where $\iota^{*} E$ and $\iota^{*} F$ are the pull-backs of $E$ and $F$ to $B_{0}$ along the source map (or the target map), and $m$ is a locally constant function defined by the order of the isotopy group of the principal stratum of a sector in $\widetilde{X}$, and $\Omega \in H^{2}(X)((\hbar))$ is the characteristic class of the star product $\star$ on $\mathcal{A}^{\hbar}$.

As the pairing between the cyclic homology and cohomology of $\mathcal{A}^{\hbar} \rtimes \mathrm{G}$ is nondegenerate, we are able to state a corollary concerning the Chern character of the element $\widehat{P(E)}-\widehat{P(F)}$ in $K_{0}\left(\mathcal{A}^{\hbar} \rtimes \mathrm{G}\right)$.

COROLLARY 3.3. Let $\mathrm{G}$ be a proper étale groupoid. The image of the Chern character $\left.C_{h}(\widehat{P(E)}-\widehat{P(F})\right)$ in $H^{\bullet}(\widetilde{X})((\hbar))$ under the map $\Psi$ defined in Theorem 2.4 is equal to the component of

$$
\frac{\hat{A}\left(B_{0} / \mathrm{G}\right) \mathrm{Ch}_{\theta}\left(\iota^{*} E-\imath^{*} F\right) \exp \left(-\frac{\iota^{*} \Omega}{2 \pi \sqrt{-1} \hbar}\right)}{\mathrm{Ch}_{\theta}\left(\lambda_{-1} N\right)},
$$

with degree less than or equal to $2 k$. We have used the same notations as in Theorem 3.2.

\subsection{AN INDEX THEOREM FOR A CONSTANT DIRAC STRUCTURE ON A 3D TORUS}

In this subsection, we look at an interesting example which is not a proper groupoid. 
We consider a 3D torus $T^{3}=S^{1} \times S^{1} \times S^{1}$, and a constant Dirac structure on $T^{3}$ defined by the following linear Dirac structure on $\mathbb{R}^{3}$,

$$
\mathcal{D}_{\alpha, \beta, \theta}=\operatorname{span}\{(\alpha, \beta, 1 ; 0,0,0),(0,-\theta, 0 ; 1,0,-\alpha),(\theta, 0,0 ; 0,1,-\beta)\} \subset \mathbb{R}^{3} \times \mathbb{R}^{3^{*}} .
$$

We remark that $\alpha, \beta, \theta$ in the above definition of $\mathcal{D}_{\alpha, \beta, \theta}$ are all constant, and that $\mathcal{D}_{\alpha, \beta, \theta}$ defines a constant Dirac structure on $T^{3}$ with the identification $T T^{3} \oplus$ $T^{*} T^{3} \simeq T^{3} \times \mathbb{R}^{3} \times \mathbb{R}^{3 *}$. We fix $\left(\theta^{1}, \theta^{2}, \theta^{3}\right)$ to be the coordinates on $T^{3}$. The Dirac structure $\mathcal{D}_{\alpha, \beta, \theta}$ defines a characteristic foliation, which is spanned by the vector field $Z=\alpha \partial_{\theta^{1}}+\beta \partial_{\theta^{2}}+\partial_{\theta^{3}}$. We assume that both $\alpha$ and $\beta$ are irrational numbers. Accordingly, the flow on $T^{3}$ generated by the vector field $Z$ is ergodic.

We consider the foliation $\mathcal{F}$ generated by $Z$ and choose a complete transversal $X=\left\{\theta^{3}=0\right\}$ to $\mathcal{F}$, which is a $2 \mathrm{D}$ torus. The holonomy group $\mathbb{Z}$ acts on $X$ by translations, mapping $\left(\theta^{1}, \theta^{2}\right)$ to $\left(\theta^{1}+\alpha, \theta^{2}+\beta\right)$. We observe that the $\mathbb{Z}$-action preserves the constant metric on $X$. Hence, the holonomy groupoid $X \rtimes \mathbb{Z}$ satisfies the conditions assumed in Section 1. Furthermore, as is explained in [23, Sec. 3], the Dirac structure $\mathcal{D}_{\alpha, \beta, \theta}$ defines a constant Poisson structure $\pi$ on $X$, i.e., $\pi=\theta \partial_{\theta^{1}} \wedge$ $\partial_{\theta^{2}}$, which is invariant under the holonomy group action. Therefore, we can apply the methods of Section 1 to construct a deformation quantization $\mathcal{A}_{X}^{\hbar} \rtimes \mathbb{Z}$ of the groupoid algebra $\mathcal{C}^{\infty}(X) \rtimes \mathbb{Z}$ (the transversal $X$ is a $2 \mathrm{D}$ torus, and there is no distinction between $\mathcal{C}^{\infty}(X)$ and $\left.\mathcal{C}_{c}^{\infty}(X)\right)$. In [23, Sec. 3] the third author with Weinstein used this idea to quantize an arbitrary constant Dirac structure on an $n \mathrm{D}$ torus. Here, our construction is slightly different as we are considering the formal deformation quantization of the smooth algebra $\mathcal{C}^{\infty}(X) \rtimes \mathbb{Z}$ instead of the $C^{*}$-algebras $C(X) \rtimes \mathbb{Z}$. We observe that $X$ has a flat connection $d$ which is symplectic with respect to the Poisson structure $\pi$ and also invariant under the $\mathbb{Z}$-action. Therefore, we choose the Fedosov connection in this example to be

$$
D=d \theta^{i} \wedge\left(\partial_{\theta^{i}}-\partial_{y^{i}}\right),
$$

where $\left(y^{1}, y^{2}\right)$ are coordinates on the Weyl algebra bundle $\mathcal{W}$. In this case, the connection 1 -form $A$ is equal to $-1 / \theta\left(y^{1} d \theta^{2}-y^{2} d \theta^{1}\right)$. The flat sections with respect to the connection $D$ defines a deformation quantization $\mathcal{A}_{X}^{\hbar}$ of $\mathcal{C}^{\infty}(X)$, and the crossed product algebra $\mathcal{A}_{X}^{\hbar} \rtimes \mathbb{Z}$ is a deformation quantization of $\mathcal{C}^{\infty}(X) \rtimes \mathbb{Z}$.

We apply Theorem 2.4 to compute the cyclic homology of the algebra $\mathcal{A}_{X}^{\hbar} \rtimes \mathbb{Z}$. Observe that the action of $\mathbb{Z}$ on $X$ is free. Therefore, the Burghelea space $B_{0}$ of the groupoid $X \rtimes \mathbb{Z}$ is equal to $X$, and the cyclic homology of $\mathcal{A}_{X}^{\hbar} \rtimes \mathbb{Z}$ is equal to the cyclic homology of the groupoid $X \rtimes \mathbb{Z}$. As the spectral sequence associated to the double complex $\Gamma_{\text {cpt }}\left(X \rtimes \mathbb{Z}_{\bullet}, \Omega^{\bullet}\right)$ degenerates at $E_{2}$, the homology of the groupoid $X \rtimes Z$ is computed as follows,

$$
\begin{aligned}
& H_{1}(X \rtimes Z ; \mathbb{C}((\hbar)))=H_{-2}(X \rtimes Z ; \mathbb{C}((\hbar)))=\mathbb{C}((\hbar)), \\
& H_{0}(X \rtimes Z ; \mathbb{C}((\hbar)))=H_{-1}(X \rtimes Z ; \mathbb{C}((\hbar)))=\mathbb{C}((\hbar))^{\times 3} .
\end{aligned}
$$


Similarly the cohomology of the groupoid $X \rtimes Z$ are computed by

$$
\begin{aligned}
& H^{0}(X \rtimes Z ; \mathbb{C}((\hbar)))=H^{3}(X \rtimes Z ; \mathbb{C}((\hbar)))=\mathbb{C}((\hbar)), \\
& H^{1}(X \rtimes Z ; \mathbb{C}((\hbar)))=H^{2}(X \rtimes Z ; \mathbb{C}((\hbar)))=\mathbb{C}((\hbar))^{\times 3} .
\end{aligned}
$$

We conclude with the following results about cyclic (co)homology of $\mathcal{A}_{X}^{\hbar} \rtimes \mathbb{Z}$.

COROLLARY 3.4. The cyclic homology and cohomology of $\mathcal{A}_{X}^{\hbar} \rtimes \mathbb{Z}$ is computed as follows,

$$
\begin{array}{ll}
H P_{0}\left(\mathcal{A}_{X}^{\hbar} \rtimes \mathbb{Z}\right)=\mathbb{C}((\hbar))^{\times 4}, & H P_{1}\left(\mathcal{A}_{X}^{\hbar} \rtimes \mathbb{Z}\right)=\mathbb{C}((\hbar))^{\times 4}, \\
H P^{0}\left(\mathcal{A}_{X}^{\hbar} \rtimes \mathbb{Z}\right)=\mathbb{C}((\hbar))^{\times 4}, & H P^{1}\left(\mathcal{A}_{X}^{\hbar} \rtimes \mathbb{Z}\right)=\mathbb{C}((\hbar))^{\times 4} .
\end{array}
$$

We write out explicit cocycles generating the cohomology of the cyclic vector space $\operatorname{Tot}^{\bullet}\left(\Omega^{\bullet}\left(B_{\bullet}\right)\right)$ for the groupoid $X \rtimes \mathbb{Z}$. As $\mathbb{Z}$ acts on $X$ freely, $B_{n}$ is a subspace of $X \times \mathbb{Z}^{\times(n+1)}$ isomorphic to

$$
\left\{\left(x, z_{0}, \ldots, z_{n}\right) \mid x \in X, z_{i} \in \mathbb{Z}, z_{0}+\cdots+z_{n}=0\right\} .
$$

Let $W^{n}$ be the delta function on $\mathbb{Z}$ supported at $n$.

(1) The degree 0 cohomology is generated by $\xi_{0}=1$, the constant function.

(2) The degree 1 cohomology is generated by $\eta_{1}=d \theta^{1} \in \Omega^{1}(X)^{\mathbb{Z}}, \eta_{2}=d \theta^{2} \in$ $\Omega^{1}(X)^{\mathbb{Z}}$ (we view $d \theta^{1}$ and $d \theta^{2}$ as differential forms supported on the unit space of the groupoid $X \rtimes \mathbb{Z}$ ), and $\eta_{3}=\delta=\sum_{n} n W^{-n} \otimes W^{n} \in \Omega^{0}\left(B_{1}\right)$ (we view $W^{-n} \otimes W^{n}$ as a function on $X \times \mathbb{Z} \times \mathbb{Z}$ taking the value 1 on $(x,-n, n)$ and 0 otherwise). We remark that although $\sum_{n} n W^{-n} \otimes W^{n}$ is an infinite sum, as a linear functional on $\left(\mathcal{C}^{\infty}(X) \rtimes \mathbb{Z}\right)^{\otimes 2}$ it is well defined because an element in $\mathcal{C}^{\infty}(X) \rtimes \mathbb{Z}$ (and also in $\mathcal{A}_{X}^{\hbar} \rtimes \mathbb{Z}$ ) has only finitely many components in $\mathbb{Z}$.

(3) The degree 2 cohomology is generated by $\xi_{1}=d \theta^{1} \wedge d \theta^{2} \in \Omega^{2}(X)^{\mathbb{Z}}, \xi_{2}=d \theta^{1} \otimes$ $\delta \in \Omega^{1}\left(B_{1}\right)^{\mathbb{Z}}$, and $\xi_{3}=d \theta^{2} \otimes \delta \in \Omega^{1}\left(B_{1}\right)^{\mathbb{Z}}$, where $\delta$ is defined as above.

(4) The degree 3 cohomology is generated by $\eta_{0}=d \theta^{1} \wedge d \theta^{2} \otimes \delta \in \Omega^{2}\left(B_{1}\right)^{\mathbb{Z}}$.

Next we compute explicit cyclic cocycles on $\mathcal{A}^{\hbar} \rtimes \mathbb{Z}$ generating $H P^{0}\left(\mathcal{A}_{X}^{\hbar} \rtimes \mathbb{Z}\right)$. (The same method can be extended easily to compute cocycles in $H P^{1}\left(\mathcal{A}_{X}^{\hbar} \rtimes \mathbb{Z}\right)$.) As has been explained above, the Fedosov connection defining $\mathcal{A}_{X}^{\hbar}$ is chosen to be $-1 / \theta\left(y^{1} d \theta^{2}-y^{2} d \theta^{1}\right)$. This simplifies the computation of the map $\Phi$ below.

We find the following generators in $H P^{0}\left(\mathcal{A}_{X}^{\hbar} \rtimes \mathbb{Z}\right)$ by inserting the connection $A$ into the definition of $\Phi$.

$$
\begin{aligned}
& \Phi\left(\xi_{0}\right) \quad\left(f W^{n}\right)= \\
& = \begin{cases}-\frac{1}{\hbar \theta} \int_{X} f d \theta_{1} \wedge d \theta_{2}, & n=0 \\
0 & \text { otherwise }\end{cases}
\end{aligned}
$$




$$
\begin{aligned}
& \Phi\left(\xi_{1}\right) \quad\left(f_{0} W^{n_{0}}, f_{1} W^{n_{1}}, f_{2} W^{n_{2}}\right)= \\
& \quad=\left\{\begin{array}{ll}
\int_{X} \tau_{2}\left(\hat{f_{0}}, \widehat{n_{0}^{*}\left(f_{1}\right)}, n_{0}^{*} n_{1}^{*}\left(f_{2}\right)\right) d \theta^{1} \wedge d \theta^{2}, & n_{0}+n_{1}+n_{2}=0 \\
0 & \text { otherwise }
\end{array} ;\right. \\
& \Phi\left(\xi_{2}\right) \quad\left(f_{0} W^{n_{0}}, f_{1} W^{n_{1}}, f_{2} W^{n_{2}}\right)= \\
& \quad=\left\{\begin{array}{ll}
\frac{\hbar}{\theta} \int_{X} n_{1} f_{0} \star n_{0}^{*}\left(f_{1}\right) \star\left(-n_{2}\right)^{*}\left(\partial_{\theta^{2}} f_{2}\right) d \theta^{1} \wedge d \theta^{2}, & n_{0}+n_{1}+n_{2}=0, \\
0 & \text { otherwise }
\end{array} ;\right. \\
& \Phi\left(\xi_{3}\right) \quad\left(f_{0} W^{n_{0}}, f_{1} W^{n_{1}}, f_{2} W^{n_{2}}\right)= \\
& \quad= \begin{cases}-\frac{\hbar}{\theta} \int_{X} n_{1} f_{0} \star n_{0}^{*}\left(f_{1}\right) \star\left(-n_{2}\right)^{*}\left(\partial_{\theta} f_{2}\right) d \theta^{1} \wedge d \theta^{2}, & n_{0}+n_{1}+n_{2}=0, \\
0 & \text { otherwise }\end{cases}
\end{aligned}
$$

where by $\hat{f}_{0}, \widehat{n_{0}^{*}\left(f_{1}\right)}, \widehat{n_{0}^{*} n_{1}^{*}\left(f_{2}\right)}$ we mean the flat sections of the Weyl algebra bundle $\mathcal{W}$ corresponding to the functions $f_{0}, n_{0}^{*}\left(f_{1}\right), n_{0}^{*} n_{1}^{*}\left(f_{2}\right)$.

We consider the following three subalgebras of $\mathcal{A}_{X}^{\hbar} \rtimes \mathbb{Z}$.

(1) The algebra $\mathcal{A}_{X}^{\hbar}$ is embedded in $\mathcal{A}_{X}^{\hbar} \rtimes \mathbb{Z}$ as functions supported at the component of zero in $X \rtimes \mathbb{Z}$.

(2) On $X$, we consider the subspace of the functions constant along $\theta_{2}$. The subspace of such functions is isomorphic to $\mathcal{C}^{\infty}\left(S^{1}\right)$, the algebra of smooth functions on $S^{1}$. The action of the group $\mathbb{Z}$ preserves this subspace, and therefore the associated crossed product algebra defines a subalgebra of $\mathcal{A}_{X}^{\hbar} \rtimes \mathbb{Z}$. This subalgebra is isomorphic to the groupoid algebra $\mathcal{C}^{\infty}\left(S^{1}\right) \rtimes_{\alpha} \mathbb{Z}$ of the transformation groupoid where $\mathbb{Z}$ acts on $S^{1}$ by translating angle $\alpha$.

(3) As in the previous case, we consider the smooth functions on $X$ constant along $\theta_{1}$, and obtain a subalgebra which is isomorphic to the groupoid algebra $\mathcal{C}^{\infty}\left(S^{1}\right) \rtimes_{\beta} \mathbb{Z}$ of the transformation groupoid of $\mathbb{Z}$ acting on $S^{1}$ by translating angle $\beta$.

We are interested in elements of $K_{0}\left(\mathcal{A}_{X}^{\hbar} \rtimes \mathbb{Z}\right)$ induced from $K_{0}$ elements associated to three subalgebras, $\mathcal{A}_{X}^{\hbar}, \mathcal{C}^{\infty}\left(S^{1}\right) \rtimes_{\alpha} \mathbb{Z}$, and $\mathcal{C}^{\infty}\left(S^{1}\right) \rtimes_{\beta} \mathbb{Z}$.

According to [8, Sec. 6.1], $K_{0}\left(\mathcal{A}_{X}^{\hbar}\right)$ is isomorphic to $K_{0}\left(\mathcal{C}^{\infty}(X)\right)$. For an element $e$ in $K_{0}\left(\mathcal{A}_{X}^{\hbar}\right) \subset K_{0}\left(\mathcal{A}_{X}^{\hbar} \rtimes \mathbb{Z}\right)$, we can compute $C h(e)$ directly using the results of [18, Sec. 5],

$$
C h(e)=\widehat{A}(X) C h(e) \exp \left(-\frac{\Omega}{\hbar}\right),
$$

where $C h(e)$ is the classical Chern character on $K_{0}\left(\mathcal{C}^{\infty}(X)\right)$ and $\Omega$ is the characteristic class of the invariant star product $\star$ on $\mathcal{A}_{X}^{\hbar}$, which in this case is $-1 / \theta d \theta^{1} \wedge$ $d \theta^{2}$. This is a differential form supported on the unit space of $X \rtimes \mathbb{Z}$.

As the consideration of subalgebras $\mathcal{C}^{\infty}\left(S^{1}\right) \rtimes_{\alpha} \mathbb{Z}$ and $\mathcal{C}^{\infty}\left(S^{1}\right) \rtimes_{\beta} \mathbb{Z}$ is a copy from one to the other, we will focus on one of them, $\mathcal{C}^{\infty}\left(S^{1}\right) \rtimes_{\alpha} \mathbb{Z}$. The 
construction in [21] defines two linearly independent elements in $K_{0}\left(\mathcal{C}^{\infty}\left(S^{1}\right) \rtimes_{\alpha}\right.$ $\mathbb{Z}) \subset K_{0}\left(\mathcal{A}_{X}^{\hbar} \rtimes \mathbb{Z}\right)$

$$
e_{1}=1, \quad e_{2}=W^{-1} g+f+g W
$$

where $f$ and $g$ are smooth functions defined on $S^{1}$ constructed in [21]. We recall the definition of $f$ and $g$. Assume that $0 \leq \alpha<1 / 2$ (there is a similar construction for $\alpha \geq 1 / 2$ ), and choose $\epsilon>0$ such that $\epsilon<\alpha$ and $\alpha+\epsilon<1 / 2$. On $[0, \epsilon]$, the function $f$ is defined to be any smooth monotone function such that $f(0)=0$ and $f(\epsilon)=1$; on $[\alpha, \alpha+\epsilon], f$ is defined by $f(x)=1-f(x-\alpha)$; on $[\epsilon, \alpha]$ define $f$ to be 1 ; and on $[\alpha+\epsilon, 1]$ define $f$ to be 0 . The function $g$ is defined to be $\left(f-f^{2}\right)^{1 / 2}$ on $[\alpha, \alpha+\epsilon]$ and zero everywhere else.

For the crossed product algebra $\mathcal{S}\left(\mathbb{Z}, \mathcal{C}^{\infty}\left(S^{1}\right)\right)$ of Schwartz functions on $\mathbb{Z}$ with values in $\mathcal{C}^{\infty}\left(S^{1}\right)$, Pimsner-Voiculescu's exact sequence proves that $K_{0}(\mathcal{S}(\mathbb{Z}$, $\left.\mathcal{C}^{\infty}\left(S^{1}\right)\right)$ ) is isomorphic to $\mathbb{Z} \oplus \mathbb{Z}$ with generators $e_{1}$ and $e_{2}$. And we can easily check that all our cyclic cocycles extend to this enlarged algebra. However, for our algebra which consists of finitely supported functions on $\mathbb{Z}$ with values in $\mathcal{C}^{\infty}\left(S^{1}\right)$, we do not have a full description of $K_{0}\left(\mathcal{C}^{\infty}\left(S^{1}\right) \rtimes_{\alpha} \mathbb{Z}\right)$, though from the computation below we know that $e_{1}$ and $e_{2}$ are different elements in $K_{0}\left(\mathcal{C}^{\infty}\left(S^{1}\right) \rtimes_{\alpha} \mathbb{Z}\right)$.

We consider the evaluation of $\Phi\left(\xi_{i}\right), i=1, \ldots, 4$, on $e_{1}$ and $e_{2}$. As $e_{1}$ and $e_{2}$ are constant along $\theta_{2}, \Phi\left(\xi_{1}\right)\left(e_{1}\right)=\Phi\left(\xi_{1}\right)\left(e_{2}\right)=\Phi\left(\xi_{2}\right)\left(e_{1}\right)=\Phi\left(\xi_{2}\right)\left(e_{2}\right)=0$. The cocycle $\Phi\left(\xi_{1}\right)$ is $-1 / \hbar \theta$ times the standard trace on $\mathcal{C}^{\infty}\left(S^{1}\right) \rtimes_{\alpha} \mathbb{Z}$. Therefore, we have

$$
\begin{aligned}
& \Phi\left(\xi_{0}\right)\left(e_{1}\right)=-\frac{1}{\hbar \theta}, \quad \Phi\left(\xi_{0}\right)\left(e_{2}\right)=-\frac{\alpha}{\hbar \theta}, \\
& \Phi\left(\xi_{3}\right)\left(e_{1}\right)=0, \quad \Phi\left(\xi_{3}\right)\left(e_{2}\right)=6 \frac{\hbar}{\theta} \int_{S^{1}} g^{2} d f\left(\theta^{1}\right)=\frac{\hbar}{\theta} .
\end{aligned}
$$

Therefore, we conclude

$$
\Psi\left(C h\left(e_{1}\right)\right)=-\frac{1}{\hbar \theta}, \quad \Psi\left(C h\left(e_{2}\right)\right)=-\left.\frac{\alpha}{\hbar \theta}\right|_{i d}+\left.\frac{\hbar}{2 \theta} d \theta_{1}\right|_{(1,-1)}-\left.\frac{\hbar}{2 \theta} d \theta_{1}\right|_{(-1,1)},
$$

where $\left.(\ldots)\right|_{(a, b)}$ means a differential form supported at the $(a, b)$ component of $B(X \rtimes \mathbb{Z})_{2}$.

Remark 3.5. We end this section with two remarks.

(1) The cocycle $\Phi\left(\xi_{3}\right)$ is closely related to the Chern character $c_{1}$ on the quantum torus algebra $\mathcal{S}\left(\mathbb{Z}, \mathcal{C}^{\infty}\left(S^{1}\right)\right)$ introduced by Connes [6]; it differs from it by a scalar because of a different normalization.

(2) The discussion in this subsection of the algebraic index theory of a constant Dirac structure on a torus can be generalized to an arbitrary constant Dirac structure on a torus of arbitrary dimension, using the ideas developed in [23] and the constructions in Section 2 of this paper. For a general constant 
Dirac structure on $T^{n}$, we can choose a complete transversal $X$ to the characteristic foliation as in [23] and consider the monodromy groupoid $G_{X}$. The unit space $G_{0}$ of the groupoid $G$ has a $G$-invariant metric, and the Dirac structure defines a $G$ invariant regular Poisson structure on $G_{0}$. All the considerations in this paper can be directly generalized to such $G_{X}$.

\section{Acknowledgements}

X. Tang's research is partially supported by NSF Grant 0703775 . H. Posthuma acknowledges support by NWO. M. Pflaum and H. Posthuma thank the Department of Mathematics of Washington University, St. Louis, MO, for hosting a research visit.

Open Access This article is distributed under the terms of the Creative Commons Attribution Noncommercial License which permits any noncommercial use, distribution, and reproduction in any medium, provided the original author(s) and source are credited.

\section{References}

1. Bressler, P., Gorokhovsky, A., Nest, R., Tsygan, B.: Deformations of algebroid stacks. (2008). arXiv:0810.0030

2. Brylinski, J.-L.: A differential complex for Poisson manifolds. J. Differ. Geom. 28(1), 93-114 (1988)

3. Brylinski, J.-L., Nistor, V.: Cyclic cohomology of étale groupoids. $K$-Theory 8, $341-$ 365 (1994)

4. Cannas da Silva, A.: Lectures on Symplectic Geometry. Lecture Notes on Mathematics, vol. 1764. Springer, Berlin (2001)

5. Connes, A.: Noncommutative Geometry. Academic Press, London (1994)

6. Connes, A.: A survey of foliations and operator algebras. In: Operator algebras and applications, Part I (Kingston, Ont., 1980). Proceedings of Symposium on Pure Mathematics, vol. 38, pp. 521-628. American Mathematical Society, Providence, R.I. (1982)

7. Crainic, M.: Cyclic cohomology of étale groupoids: the general case. $K$-Theory 17, 319-362 (1999)

8. Fedosov, B.: Deformation Quantization and Index Theory. Academic Press, London (1995)

9. Fedosov, B. : On $G$-trace and $G$-index in deformation quantization. Conference Moshé Flato 1999 (Dijon). Lett. Math. Phys. 52(1), 29-49 (2002)

10. Feigin, B., Felder, G., Shoikhet, B.: Hochschild cohomology of the Weyl algebra and traces in deformation quantization. Duke Math. J. 127(3), 487-517 (2005)

11. Getzler, E., Jones, J.D.: The cyclic homology of crossed product algebras. J. Reine Angew. Math. 445, 161-174 (1993)

12. Khalkhali, M., Rangipour, B.: On the generalized cyclic Eilenberg-Zilber theorem. Can. Math. Bull. 47(1), 38-48 (2004)

13. Loday, J.-L.: Cyclic Homology. Grundlehren der mathematischen Wissenschaften, vol. 301. Springer, Berlin (1998)

14. Molino, P.: Riemannian Foliations. Birkhäuser, Boston (1988) 
15. Nest, R., Tsygan, B.: Algebraic index theorem. Commun. Math. Phys 172, 223-262 (1995)

16. Neumaier, N., Pflaum, M.J., Posthuma, H., Tang, X.: Homology of formal deformations of proper étale Lie groupoids. J. Reine Angew. Math. 593, 117-168 (2006)

17. Pflaum, M.J., Posthuma, H., Tang, X.: An algebraic index theorem for orbifolds. Adv. Math. 210, 83-121 (2007)

18. Pflaum, M.J., Posthuma, H., Tang, X.: Cyclic cocycles on deformation quantizations and higher index theorems. (2008). arXiv:0805.1411

19. Pflaum M.J., Posthuma H., Tang X., Tseng H.-H.: Orbifold cup products and ring structures on Hochschild cohomologies. (2007). arXiv:0706.0027

20. Phillips N.CH.: Equivariant $K$-theory for proper actions. Pitman Research Notes in Mathematics Series, vol. 178. Longman Scientific \& Technical, Harlow (copublished in the United States with Wiley, New York) (1989)

21. Rieffel, M.A.: $C^{*}$-algebras associated with irrational rotations. Pac. J. Math. 93(2), 415-429 (1981)

22. Tang, X.: Deformation quantization of pseudo Poisson groupoids. Geom. Funct. Anal. 16(3), 731-766 (2006)

23. Tang, X., Weinstein, A.: Quantization and Morita equivalence for constant Dirac structures on tori. Ann. Inst. Fourier (Grenoble) 54(5), 1565-1580 (2004)

24. Weibel, C.H.: An Introduction to Homological Algebra. Cambridge Studies in Advanced Mathematics, vol. 38. Cambridge University Press, London (1995) 